\title{
CANTOR SETS IN METRIC MEASURE SPACES
}

\section{BERNARD R. GELBAUM ${ }^{1}$}

The discussions and results below are the fruits of a number of conversations the author held with Harry Furstenberg, Richard Darst and Robert Zink. The burden of the paper is the phenomenon that may be paraphrased (approximately) as follows: Every compact metric measure space is the union of the Cantor set and an open set of small measure. The preceding sentence is false. Its correct version is the theorem below.

We preface the argument with the following remarks, many of which, despite their elementary nature, are made for completeness.

a. A compact metric space $X$ is separable. It is either countable or its cardinality card $(X)$ is $\mathfrak{c} \equiv \operatorname{card}(R)$. If card $(X)=\mathfrak{c}$, then $X$ contains a homeomorphic image of the Cantor set [1].

b. If $S$ is the $\sigma$-ring generated by the compact sets of the compact metric space $X$, and if $\mu$ is a finite nonatomic measure defined for the elements of $S$, then $\mu$ is regular [2]. In what follows, we assume that $X$ is a compact metric space, that $\mu$ is a nontrivial, finite nonatomic measure defined for the elements of $S$. Thus $\operatorname{card}(X)=\mathfrak{c}$ and $\mu$ is regular. The next result was suggested by Darst. The notations above prevail.

Lemma 1. There is a countable neighborhood basis $u=\left\{U_{n}\right\}$ for the topology of $X$ and such that $\mu\left(\partial U_{n}\right)=0$ for all $U_{n} \in \mathcal{U}$, where $\partial U_{n}$ denotes the boundary of $U_{n}$.

Proof. Let $\rho$ be the metric for $X$ and let $S_{x . d}=\{y: \rho(x, y)<d\}$, for $d>0, x \in X$. For each $x$, the boundaries $\partial_{x, d} \equiv \partial S_{x, d}$ are disjoint for distinct $d: d_{1} \neq d_{2}$ implies $\partial_{x, d_{1}} \cap \partial_{x, d_{2}}=\varnothing$. Since $\mu$ is finite, for only countably many $d$ is $\mu\left(\partial_{x, d}\right)>0$. For each $x$ let $S_{x, d_{n}(x)}$ be such that $0<d_{n+1}(x)<d_{n}(x), d_{n}(x) \rightarrow 0$ as $n \rightarrow \infty$ and such that $\mu\left(\partial_{x, d_{n}(x)}\right)=0$. For each $m=1,2, \cdots$, let $d_{n_{m}}(x)$ be the first $d_{n}(x)$ under $1 / m: d_{n_{m}}(x)$ $=\sup \left\{d_{n}(x): d_{n}(x)<1 / m\right\}$. Then for each $m$ there is a finite cover $\left\{S_{x_{m j}, d_{n_{m}}\left(x_{m j}\right)}: j=1,2, \cdots, J_{m}\right\}$ of $X$. Let $\mathcal{u}$ be the set of all

$$
S_{x_{m j}, d_{n_{m}}\left(x_{m j}\right)}, \quad m=1,2, \cdots, j=1,2, \cdots, J_{m} .
$$

Then $\mathcal{u}$ is a countable family of open sets, the sets of $u$ constitute a cover for $X$ and, as we now show, $u$ is a basis for the topology of $X$.

Received by the editors April 24, 1969 and, in revised form, July 28, 1969.

1 The author thanks the National Science Foundation for its grant GP 8291, one of several sources of support for the research reported herein. 
Indeed, if $x \in X$ and if $V$ is an open set containing $x$, let $S_{x, d} \subset V$. Then let $2 / m<d$ and choose $S \equiv S_{x_{m j}, d_{n_{m}}\left(x_{m j}\right)}$ containing $x$. Then since the diameter of $S$ does not exceed $2 / m$ we see that $x \in S \subset S_{x, d} \subset V$. Thus $u$ is a countable basis for the topology of $X$.

Lemma 2. If $Y$ is a Hausdorff space, if $u=\{U\}$ is a neighborhood basis for $Y$ and if $V_{U}$ is an open set containing $\partial U$ for each $U$, then $Y \backslash \bigcup_{\mathcal{U}} V_{U}$ is either empty, a one-point set or totally disconnected.

Proof. If $Y \backslash \bigcup_{\mathfrak{u}} V_{U}$ contains more than one point, let $Z$ be a connected subset of $Y \backslash \bigcup_{\mathcal{U}} V_{U}$, where card $(Z)>1$. Then since $Y \backslash \bigcup_{\mathfrak{U}} V_{U}$ is closed, we see that we may assume $Z$ is closed. Choose a point $z$ in $Z$ and a neighborhood $U \in \mathcal{U}$ such that $Z \backslash U \neq \varnothing$. Then $\varnothing \subset\{z\}$ $\subset Z \cap\left(U \backslash V_{U}\right)=Z \cap U$ since $Z \subset Y$, and thus $Z=(Z \backslash U) \cup(Z \cap U)$, the union of two disjoint nonempty sets. Of course, $Z \backslash U$ is closed. However, $\bar{U} \backslash V_{U}=U \backslash V_{U}$ since if $x \in \bar{U} \backslash V_{U}$ and if $x \in U$, then $x \in \partial U$, whence $x \in V_{U}$ whence $x \in \bar{U} \backslash V_{U}$. Thus $Z \cap U=Z \cap\left(\bar{U} \backslash V_{U}\right)$ is closed and so $Z$ cannot be connected.

TheOREM. Let $X$ be a compact metric space; let $\mu$ be a nontrivial, nonatomic, finite measure defined on the Borel sets of $X$. For each $\epsilon>0$ there is an open set $W$ such that $(X \backslash W)$ is homeomorphic to the Cantor set and $\mu(W)<\epsilon$.

Proof. Let $\left\{U_{n}\right\}$ be a countable basis that is a subset of $u$. Since $\mu$ is regular we may choose an open set $V_{n} \supset \partial U_{n}$ and such that $\mu\left(V_{n}\right)$ $<\epsilon / 2^{n}, n=1,2, \cdots$. Then $X \backslash \bigcup_{n} V_{n}$ is totally disconnected, compact and hence, by the Cantor-Bendixson theorem [3], the union of a perfect set $P$ and a countable set $D$. We may assume $\mu\left(X \backslash \bigcup_{n} V_{n}\right)>0$ whence $P$ is nonempty and thus $P$ and $C$ are homeomorphic [1]. The open set $W=X \backslash P$ has measure less than $\sum_{n} \mu\left(V_{n}\right)+\mu(D)<\epsilon$.

Note. Owing to the prefatory remarks, we can conclude that if card $(X)=\mathrm{c}$, then $X$ contains a homeomorphic image $P$ of the Cantor set on which there lives a finite measure $\mu$. In this instance $\mu(X \backslash P)$ $=0$. The impact of the theorem is that if the measure $\mu$ is given a priori, then for some $P, \mu(X \backslash P)<\epsilon$.

In a forthcoming paper [4], Oxtoby, dealing with related topics, shows that the theorem above conjoined with a result in [2] yields:

THEOREM. Let $X$ be a complete separable metric space, let $\mu$ be a nonatomic Borel measure in $X$, and let $B$ be a Borel set with $0<\mu(B)<\infty$. For each $\epsilon>0$ there is a set $C \subset B$ such that $C$ is homeomorphic to the Cantor set and $\mu(B \backslash C)<\epsilon$. 
Proof. By [2, p. 183, (3e)] there exists a compact set $K \subset B$ such that $\mu(K)>\mu(B)-\epsilon$. By the above theorem, $K$ contains a set $C$ homeomorphic to the Cantor set with $\mu(C)>\mu(B)-\epsilon$.

\section{REFERENCES}

1. P. Alexandroff and H. Hopf, Topologie, Springer, Berlin, 1935; reprint Chelsea, New York, 1965. MR 32 \#3023.

2. P. Halmos, Measure theory, Van Nostrand, Princeton, N. J., 1950. MR 11, 504.

3. K. Kuratowski, Topologie. Vol. I, PWN, Warsaw, 1933, English transl., Academic Press, New York and PWN, Warsaw, 1966. MR 36 \#839; MR 36 \#840.

4. J. C. Oxtoby, Homeomorphic measures in metric spaces, Proc. Amer. Math. Soc. (to appear).

University of California, Irvine 